\title{
Essais
}

Revue interdisciplinaire d'Humanités

$12 \mid 2017$

Textes et contextes : entre autonomie et dépendance

\section{Texte et contexte en dialogue}

Denis Vernant

\section{(2) OpenEdition}

Journals

Édition électronique

URL : http://journals.openedition.org/essais/2673

DOI : 10.4000/essais. 2673

ISSN : 2276-0970

Éditeur

École doctorale Montaigne Humanités

Édition imprimée

Date de publication : 15 juin 2017

Pagination : $33-50$

ISBN : 979-10-97024-02-4

ISSN : 2417-4211

Référence électronique

Denis Vernant, "Texte et contexte en dialogue », Essais [En ligne], 12 | 2017, mis en ligne le 12 octobre 2020, consulté le 25 octobre 2020. URL : http://journals.openedition.org/essais/2673 ; DOI : https:// doi.org/10.4000/essais. 2673 


\section{Texte et contexte en dialogue}

\section{Denis Vernant}

«Il faut être deux .../... pour comprendre un ciel bleu, pour nommer une aurore. »

Gaston Bachelard, Préface à Je et Tu.

Les notions de texte et de contexte relèvent pour une large part du discours familier. D'où leurs fortes polysémie et ambivalence. Elles requièrent donc un effort tout particulier de conceptualisation. Mais toute conceptualisation suppose un ancrage théorique qui l'autorise. Or texte et contexte peuvent se conceptualiser de façons fort différentes. Le texte constitue le concept majeur et l'objet premier de la théorie littéraire et de l'approche sémantique comme narrative. Quant au contexte, il caractérise généralement toute approche pragmatique. D'où une délicate plurivalence conceptuelle. Il importe donc de déterminer $a b$ initio le paradigme et le cadre théorique dans lesquels on situe l'analyse.

Nous proposons dans ce qui suit d'examiner les relations entre texte et contexte en les faisant " entrer en dialogue ». Nous le ferons en recourant à notre conception du dialogue s'inscrivant dans le paradigme actionnel qui depuis le début du $\mathrm{XX}^{\mathrm{e}}$ siècle aborde les questions langagières en termes d'interactions discursives et dialogiques (dimension pragmatique) indexées sur des transactions effectives entre agents dans un monde partagé (dimension praxéologique $)^{1}$. Il s'agira donc d'aborder les rôles du texte et du contexte au cours du procès dialogique d'échange discursif. Dans un tel cadre théorique, nous serons conduit à distinguer le Discours du Texte, puis, en nous concentrant sur la seule dimension discursive, à définir et articuler texte, cotexte, intexte, contexte, situation et arrière-plan en établissant leur rôle dans le déploiement progressif du sens, de la compréhension et de la communication.

1 Voir notre article " Le paradigme actionnel en philosophie du langage ", Entre connaissance et organisation : l'activité collective, R. Teulier et Ph. Lorino (éds), Paris, Éd. de la Découverte, coll. Recherche, 2005, p. 25-53. 


\section{Discours/Texte}

Sans remonter aux Mégariques ni même aux racines récentes du dialogisme chez Buber, Bakhtine et Jacques ${ }^{2}$, partons de la leçon bien connue de Benveniste selon laquelle le Discours est dialogue qui fonde le jeu des personnes dans et par l'allocution :

C'est dans et par le langage que l'Homme se constitue comme sujet; parce que le langage seul fonde en réalité, dans $s a$ réalité qui est celle de l'être, le concept d'« ego »..../... Est « ego » qui dit « ego ». Nous trouvons là le fondement de la "subjectivité ", qui se détermine par le statut linguistique de la "personne ». La conscience de soi n'est possible que si elle s'éprouve par contraste. Je n'emploie $j e$ qu'en m'adressant à quelqu'un, qui sera dans mon allocution un $t u$. C'est cette condition de dialogue qui est constitutive de la personne, car elle implique en réciprocité que je deviens $t u$ dans l'allocution de celui qui à son tour se désigne par $j e^{3}$.

En tant qu'actualisation effective de la langue dans un échange communicationnel oral, nous définissons le Discours comme une activité conjointe, un processus ouvert et créatif résultant de l'interaction langagière entre (au moins) deux interlocuteurs ${ }^{4}$. Bref, il s'agit en termes aristotéliciens d'une praxis possédant sa fin en elle-même $e^{5}$ Au Discours ainsi conceptualisé comme échange de paroles vives au cours d'un événement temporel on peut opposer frontalement le Texte comme résultat d'une action poïétique de production d'un écrit clos sur lui-même. Ce Texte s'avère ainsi une œuvre (littéraire si au scripteur est reconnu le statut non d'écrivant mais d'écrivain) achevée au terme de l'action de production et qui au moment même de sa rédaction n'est pas soumise à l'irrémédiable du flux temporel ("ce qui est dit, est dit»), puisque sont toujours possibles ratures, reprises, ajouts, suppressions, modifications, etc. Fruit d'un auteur, une telle œuvre s'autonomise et ouvre au lecteur potentiel de multiples interprétations et analyses relevant de la narratologie. Il convient donc de distinguer nettement ce qui relève de la discursivité dialogique (Sprachlichkeit) et ce qui dépend de l'écriture et la textualité (Schriftlichkeit) ${ }^{6}$ :

Il ne suffit pas de dire que la lecture est un dialogue avec l'auteur à travers son ouvre ; il faut dire que le rapport du lecteur au livre est d'une tout autre nature; le dialogue est un échange de questions et de réponses; il n’y a pas

2 Pour un rappel, consulter notre Introduction à la philosophie contemporaine du langage, Paris, A. Colin, 2010, $\$ 6$, p. 129-133.

3 Problèmes de linguistique générale, I, Paris, Gallimard, Coll. Tel n 7, 1966, chap. XXI : " De la subjectivité dans le langage ", p. 260.

4 Pour une analyse précise, voir notre Du Discours à l'action, études pragmatiques, Paris, PUF, 1997, chap. V, p. 87-106.

5 Éthique à Nicomaque, L. I, chap. 1, 1094 a.

6 Le fait qu'un texte donné prenne forme dialogale, tel un «dialogue » de Platon, les Discorsi de Galilée, le Dialogue de Leibniz, etc., ne change en rien son caractère non dialogique : c'est un Texte, artéfact résultant du procès d'écriture. 
d'échange de cette sorte entre l'écrivain et le lecteur ; l'écrivain ne répond pas au lecteur ; le livre sépare plutôt en deux versants l'acte d'écrire et l'acte de lire qui ne communiquent pas; le lecteur est absent à l'écriture ; l'écrivain est absent à la lecture. .../... [le texte] se substitue à la relation de dialogue qui noue immédiatement la voix de l'un à l'ouïe de l'autre?

\section{L'apport du texte}

Adoptant d'emblée une perspective discursive et dialogique, nous n'aurons pas ici affaire au Texte ainsi conceptualisé. Pour autant, nous pourrons parler de texte en un autre sens : comme simple trace écrite, transcription scripturaire, verbatim, recueil brut des paroles échangées au cours du déroulement du discours, c'est-à-dire du dialogue oral, effectif, de face à face ${ }^{8}$.

Considéré comme simple trace écrite de ce qui fut successivement dit, ce texte n'autorise selon nous qu'une analyse sémantique du contenu des énoncés, abstraction faite de toute dimension dialogique. Ainsi dans le texte du fameux dialogue de Molière l'énonciation "Le petit chat est mort " n'est interprétée que sémantiquement et au sens propre de l'indication d'un chat (que les deux interlocuteurs connaissent) auquel il est attribué le fait d'être petit et désormais mort. Bref, ainsi "pris au pied de la lettre » le texte ne nous fournit qu'une liste d'énoncés dont on décode la signification sémantique. C'est manifestement peu, mais c'est le point de départ obligé de la construction dialogique progressive du sens pragmatique à partir du décodage des signifiants sémantiques.

\section{Le rôle du cotexte}

Au texte il convient d'ajouter le cotexte. Plus exactement, il n'est en rien nécessaire d'ajouter quelque chose au texte dans sa matérialité, mais simplement de prendre en compte cette fois les énonciations au cours du procès dialogique. Il s'agit alors non plus de considérer les énoncés isolés, mais les énonciations en tenant compte de leur places et rôles dans la succession processuelle. Le cotexte n'est autre que le déploiement du texte au fil du déploiement dialogique.

Une telle prise en considération du cotexte permet d'abord les désambigüsations sémantiques. Considérons un titre d'un reportage qui commence par :

Une grue dans les rues de Manhattan.

7 Paul Ricœur, Du texte à l'action, II, 1 : «Qu'est-ce qu'un texte », Paris, Seuil, Essais, 1986, p. 155.

8 Bien entendu, en dehors de cette situation canonique existent d'autres modalités dialogiques. D'abord, les échanges oraux à distance, tels ceux téléphoniques ou par visio-conférence. Ensuite, ceux recourant au médium écrit, comme les SMS, forums internet ou réseaux sociaux. Alors, le texte s'écrit au fur et à mesure des échanges. Mais même dans ce dernier cas de figure, on reste dans le champ de la dialogicité. Dans les deux cas l'analyse ne requiert que des adaptations pour rendre compte de la spécificité et des contraintes du médium.

9 L'École des femmes, Acte 2, scène 5. 
On ne saurait l'interpréter sémantiquement qu'à la lumière de la suite :

Le 5 février 2016 à 8h24 une grue géante s'est effondrée en plein cœur de New York.

Le nom " grue " admettant en Français au moins trois significations, seul le cotexte immédiat peut permettre de trancher.

Ainsi le décodage sémantique des énonciations dépend-il de la temporalité processuelle du dialogue. Et, par exemple, l'interprétation d'une énonciation au début du dialogue peut être modifiée rétroactivementà la lumière de la suite du dialogue :

Au cours d'une conversation quelqu'un me dit hors de propos : «Je te souhaite bonne chance ". Cela m'étonne ; mais plus tard, je m’aperçois que ces mots ont un lien avec ce qu'il pense à mon sujet. Et dorénavant ils ne me paraissent plus dénués de sens ${ }^{10}$.

Outre cet apport sémantique, le cotexte joue un rôle pragmatique crucial en ce qu'il permet d'appréhender le déroulement effectif du dialogue dans sa temporalité intrinsèque. Dès lors, on peut assigner une fonction dialogique propre à chaque énonciation corrélativement à sa place dans le procès dialogique. Par exemple, prennent alors sens pragmatique les procédures de répétition et d'écho.

Le fait pour un interlocuteur donné de se répéter peut signifier une incompréhension du partenaire et donc une menace pour la poursuite du dialogue. Bien souvent, s'ouvre alors une incidence métadiscursive ${ }^{11}$ pour y remédier. C'est par exemple le cas dans le dialogue informatif suivant :

1 - Je voudrais l'adresse d'un taxidermiste.

2 - Que signifie taxidermiste ?

3 - Pardon!

4 - Que veut dire taxidermiste?

5 - Vous voulez savoir ce que signifie taxidermiste?

6 - Oui.

7 - C'est quelqu'un qui empaille les animaux.

8 - Voici une adresse : ... ${ }^{12}$.

En 2 et 4 le répondant est conduit à répéter à l'identique sa question. Une telle répétition initiale manifeste une incompréhension dont témoigne l'interjection en 3.

De même l'écho consiste pour un locuteur à reprendre (intégralement ou non) l'énonciation antérieure de son allocutaire. Selon le cotexte, cela peut

10 Wittgenstein, De la certitude, trad. fr. J. Fauve, Paris, Gallimard, Coll. Tel, 1965, $\$ 469$.

11 Sur le rôle important du métadiscursif, voir notre Discours et Vérité, Analyses pragmatique, dialogique et praxéologique de la véridicité, Paris, Vrin, 2009, chap. IV : "Considération et acte de citer ".

12 Daniel Luzzati, Recherches sur le dialogue homme-machine, modèles linguistiques et traitements automatiques, thèse d'État sous la direction de Mary-Annick Morel, Paris III, juin 1989, p. 377. 
produire un renforcement pragmatique. Ainsi dans le dialogue précédent, la reprise en écho en 5 de 2 met fin à l'incompréhension précédente et 7 y apporte remède par une définition relevant d'une montée métadiscursive ${ }^{13}$.

Mais dans un autre cotexte, le fait de reproduire systématiquement l'écho de l'allocutaire provoque un psittacisme qui peut constituer une forme particulière de refus déguisé du dialogue. Ainsi d'Agnès qui ne prisant guère le commerce d'Arnolphe produit initialement deux répliques en écho.

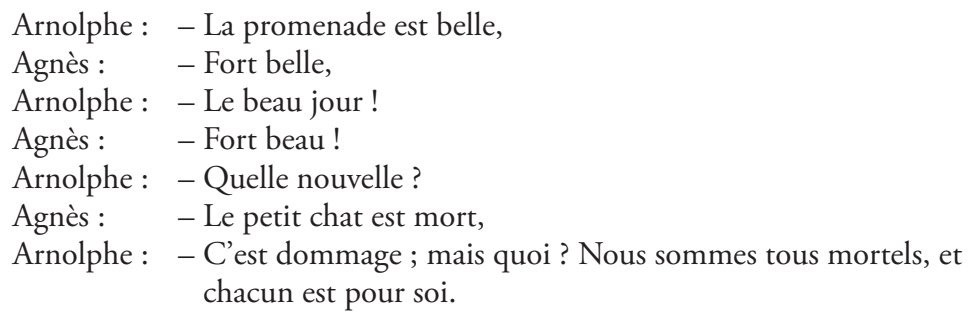

Plus généralement, la considération du cotexte global du dialogue permet seule d'assigner fonction dialogique aux interventions. Par exemple à la suite d'une question posée peuvent succéder un silence, une réponse, une réplique, une mise en cause ou même une mise en question, toutes fonctions qui dépendent de l'impact dialogique de l'énonciation au moment précis du dialogue ${ }^{14}$. Ainsi, dans le dialogue moliéresque l'énonciation d'Agnès " Le petit chat est mort " peut être interprétée non plus, comme précédemment, comme une simple réponse à une question fournissant une information, mais bien comme une réplique insidieuse consistant à fuir le dialogue effectif en relatant un fait anodin ne prêtant pas à discussion ${ }^{15}$.

Ainsi, seul le cotexte peut assurer la fonction dialogique des énonciations proférées au cours du dialogue. Bien entendu, interviennent aussi d'autres considérations relevant cette fois du contexte et de la situation, mais c'est bien le cotexte qui fournit les données initiales.

\section{L'intérêt de l'intexte}

Les données de surface de tout dialogue oral effectif sont composées des interactions langagières produites. Ces interactions verbales se présentent sous forme d'échanges de mots fournis par le code de la langue partagée. Le jeu dialogique s'apparente alors à un ping-pong informationnel. Mais ce n'est là que le plus convenu et non nécessairement le seul et surtout le plus important

13 Pour une analyse plus précise selon notre modèle projectif du dialogue informatif, voir $D u$ discours à l'action, chap. VI, p. 112-125.

14 Sur la définition de ces fonctions dialogiques, voir Du Discours à l'action, chap. VII.

15 Reste que comme nous avons en fait affaire à un Texte de théâtre, une autre interprétation est toujours possible qui ferait d'Agnès une simple ingénue! 
des échanges. Il convient donc d'entendre « langagier " en un sens bien plus large que verbal qui doit inclure aussi (et surtout dans certains types de dialogues) les signes, signaux et indices que constituent les intonations, le rythme de parole, les regards, les mimiques, les gestes, les postures, les attitudes, les tremblements, le fait de rougir, de suer, etc. Un tel intexte ${ }^{16}$ non verbal peut faire l'objet d'analyses précises et circonstanciées ${ }^{17}$. Notons simplement qu'il suppose non un décodage de signes de la langue, mais une lecture de signaux qui mettent en jeu le corps. Certaines données intextuelles ont une fonction quasi automatique, tels les échanges de regards qui commandent les prises de parole successives. Mais d'autres peuvent selon les types de dialogues et leurs objectifs jouer un rôle positif ou négatif. Par exemple, un rougissement marqué après une intervention durant un dialogue technique de conception peut avoir un effet parasite, voire perturbateur (d'où l'éreutophobie). À l'inverse, au cours d'un dialogue de séduction la lecture automatique de la dilatation pupillaire (mydriase) du/de la partenaire fournit une information subconsciente qui fait avancer positivement le dialogue.

Pour l'essentiel, les données intextuelles mettent en jeu ce que nous appelons les transactions intersubjectives, c'est-à-dire non plus les informations objectivement échangées, mais les relations vécues et incarnées au cours du dialogue par (au moins) deux partenaires qui se co-constituent comme tels. On retrouve ici la leçon platonicienne selon laquelle il ne saurait y avoir d'érotétique sans érotique. Ce qui, dans notre vocabulaire, signifie que l'interaction langagière, considérée dans l'ensemble de ses dimensions, est soumise aux contraintes transactionnelles intersubjectives.

Ainsi l'intexte n'a pas le caractère secondaire du paratexte en théorie de l'écriture : il constitue au contraire la chair même du texte et témoigne du caractère multimodal du dialogue. Mettant face à face deux interlocuteurs, le dialogue ne saurait en rien se limiter à un échange verbal; il peut même se passer de mots. Ainsi, dans la phase phatique initiale d'ouverture d'un dialogue une salutation, une poignée de main peuvent accompagner et confirmer un «bonjour » ou bien en tenir lieu. Des actions non verbales conventionnalisées peuvent parfaitement valoir comme actes de discours tel l'exemple austinien de l'action de "lancer une tomate dans une réunion politique ${ }^{18}$ qui constitue un authentique geste de discours.

16 Nous utilisons ce terme pour ne pas autoriser de confusions avec les concepts de para-, péri-, épitexte relevant de la théorie de l'écriture et non de la dialogicité. En imprimerie, le in texte vient illustrer le texte en s'incrustant dedans.

17 Pour une présentation générale, voir Jacques Cosnier, "Le gestes du dialogue ", La Communication, état des savoirs, P. Cabin et J.-F. Dortier (éds), Éditions Sciences Humaines, 2008, p. 119-128.

18 Quand dire, c'est faire, trad. fr. G. Lane, Paris, Seuil, 1970, neuvième conférence, p. 120, note** et dixième conférence, p. 129. Voir aussi le geste napolitain de mépris opposé par Piero Sraffa à la conception logiciste du langage du premier Wittgenstein, vide Ray Monk, Wittgenstein, le devoir de génie, trad. fr. A. Gerschenfeld, Paris, Flammarion, 2009, p. 258. 


\section{Du contexte}

Dans son usage relâché la notion de contexte est quasiment synonyme de pragmatique. Les données contextuelles fourniraient de quoi passer de la signification sémantique au sens pragmatique ${ }^{19}$ :

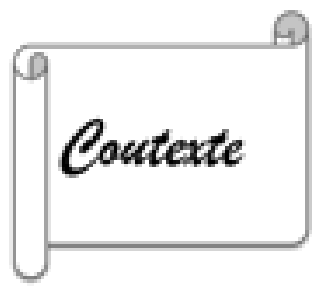

Fig. 1 : Influence du contexte.

En cette acception, tout ce que nous allons aborder maintenant relèverait $\mathrm{du}$ contexte lato sensu ${ }^{20}$. Toutefois notre approche analytique ne saurait se satisfaire d'un tel usage et nous proposons de définir le concept de contexte de façon précise et plus étroite. Appelons donc contexte stricto sensu le seul ensemble des textes qui recensent les énonciations produites au cours de dialogues antérieurs ayant eu effectivement lieu entre les partenaires du dialogue. C'est donc l'historique des dialogues précédents, le texte des échanges passés qui en même temps pèse sur et éclaire le sens et la finalité du présent dialogue.

Bien souvent, une telle dimension historique est négligée puisque l'on ne prend en compte que le dialogue en cours. Mais la plupart du temps un dialogue s'instaure entre partenaires qui se connaissent en ce qu'ils ont déjà conversé, échangé. Le dialogue prend alors place dans une série de dialogues qui le contraignent plus ou moins fortement. Ainsi importe-t-il de prendre en compte la répétition des jeux dialogiques. Ce que font Caelen et Xuereb en recourant à la théorie des jeux :

Dans de nombreux cas (presque la plupart du temps), le dialogue s'inscrit dans une pratique générale répétitive : le dialogue devient un jeu répété ; les interlocuteurs ont une réputation, un degré de confiance a priori, des contraintes externes au dialogue et des héritages des parties antérieures. On appliquera la théorie des jeux répétés à horizon infini pour mettre en évidence des jeux

19 Dans la fig. 1 " Coutexte ", écrit en Brush Script MI italic 44, se lit "Contexte ".

20 En ce sens large, le contexte est définissable comme l'entour [l'ancien envirum] de l'élément faisant l'objet d'une analyse d'un certain type. Pour l'analyse sémantique, le contexte du mot est la phrase dans et par laquelle il acquiert signification. Voir le Principe du contexte de Gottlob Frege : "On doit rechercher ce que les mots veulent dire non pas isolément mais pris dans leur contexte phrastique " (traduction complétée : nach der Bedeutung der Wörter muß im Satzszusammenhange, nicht in ihrer Vereinzelung gefragt werden), Les Fondements de l'arithmétique : recherche logico-mathématique sur le concept de nombre, trad. fr. Cl. Imbert, Paris, Seuil, 1969, p. 122. À noter que le vocable allemand présente le mérite de ne pas évoquer la textualité et d'insister sur le caractère relationnel (zusammen) de l'analyse contextuelle. 
de confiance et de pouvoir en œuvre dans ce type de dialogue. Ce sont par exemple les discussions client/fournisseur ou patron/employé, ou encore les discussions à la cafétéria dans une entreprise. De ces dialogues répétés, le gain conjoint est hérité des dialogues précédents, il donne le " climat psychologique " au début du dialogue, puis ce climat sera modifié par le comportement des interlocuteurs au cours du dialogue ${ }^{21}$.

Considérons un de leurs exemples :

Vendeur : - Alors la petite dame comment ça va aujourd'hui ? J'ai de belles courgettes aujourd'hui, toutes fraîches.

Cliente : - Ça va bien et vous? Combien vos courgettes ? C'est pas trop de saison ça, dites-moi...

Vendeur: - C'est pas cher pour vous, je vous fais un prix, vous êtes belle comme tout aujourd'hui.

Cliente : - Merci, vous êtes gentil [... acte d'achat...]. À demain.

La semaine précédente, le marchand de légumes a vendu des aubergines à la cliente. Son objectif est de lui vendre maintenant des courgettes, mais surtout de la fidéliser en prévision de prochaines transactions. D'où son ton familier et enjôleur. La cliente, qui n'est pas dupe, prévoit en échange un rabais. La négociation réussit car « Le vendeur fait une concession sur le prix, il baisse son gain immédiat pour augmenter son gain espéré (sur les ventes futures) $»^{22}$. Il est manifeste ici que le dialogue ne prend sens pragmatique et finalité praxéologique que resitué dans une série qui lie dialogiquement les deux partenaires.

\section{La nécessaire prise en compte de la situation}

Comme le texte et le cotexte, le contexte ainsi conçu relève des interactions langagières entre les partenaires du dialogue. Ces différentes formes de textes fournissent de précieuses informations pour comprendre ce qui se passe lors de l'échange dialogique. Mais celles-ci ne sauraient aucunement suffire pour saisir le sens et la finalité de ce qui se joue dans et par le dialogue. Comme activité conjointe, le dialogue ne saurait se limiter à sa seule dimension interactionnelle. Récusant toute clôture communicationnelle d'ordre idéaliste, il convient de se souvenir de la remarque wittgensteinienne selon laquelle " tout jeu de langage (Sprachspiel) est subordonné à une forme de vie (Lebensform) $»^{23}$. Dans notre vocabulaire, cela revient à dire que les interactions langagières sont tributaires des transactions intersubjectives et intramondaines qui leur donnent sens, ce que nous schématisons ainsi ${ }^{24}$ :

21 "L'altérité dans le dialogue ", Interactions et Intercompréhension : une approche comparative, sous la direction de Marine Grandgeorge, Brigitte Le Pévédic, Frédéric Pugnières-Saavedra, Collectif des Éditeurs Indépendants, Paris, 2013, \$6.

22 Pour une analyse formelle, voir Caelen \& Xuereb, «Dialogue et théorie des jeux », \$ VIII.

23 Investigations philosophiques, 1989, $\$ 23$.

24 Pour le détail de l'analyse, voir Du Discours à l'action, chap. V. 


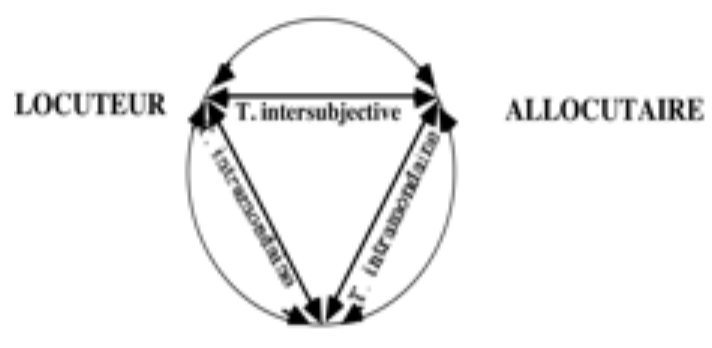

Image du / MONDE

Fig. 2 : Dimensions inter-et transactionnelles du dialogue.

Dans sa dimension pragmatique, le dialogue se présente d'abord comme une interaction langagière (représentée dans la fig. 2 par les arcs) qui instaure une relation communicationnelle entre locuteur et allocutaire et qui leur permet, en négociant des interactes ${ }^{25}$, de construire une image du monde sanctionnant leur accord sur les objets et le micro-monde qu'ils vont partager. On se situe là dans la dimension représentationnelle d'un échange complexe de signes et signaux divers fournis par les texte, cotexte, intexte et contexte. Mais dans la mesure où nous considérons que le dialogue constitue un phénomène fondamentalement hétéronome, il convient de compléter l'analyse pragmatique de l'interaction langagière par un examen praxéologique ${ }^{26}$ de sa dimension transactionnelle (que, dans le dessin ci-dessus, figure le triangle central).

Nous convenons d'appeler situation ce contexte spécifiquement praxéologique qui se déploie en ses deux dimensions intersubjectives et intramondaines. Une telle situation est d'abord caractérisée dans sa singularité dialogique par le jeu des déictiques qui en précise les circonstances: allocutaires, lieu, temps, objets délocutés ${ }^{27}$.

25 Sur la définition des interactes comme co-constructions par les interlocuteurs et de leurs différentes fonctions dialogiques (par ex. de réponse, réplique ou mise en cause), voir $D u$ Discours à l'action, chap. VIII.

26 Rappelons que la praxéologie, ou théorie générale de l'action, a été inventée par le français Alfred Espinas en 1897.

27 Benveniste, Problèmes de linguistique générale, Paris, Gallimard, Coll. Tel, 1974, II, chap. V, p. $79-88$. 


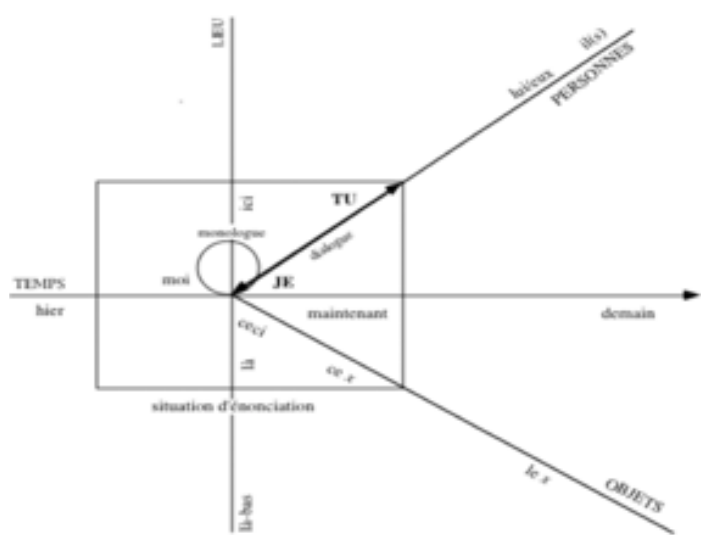

Fig. 3 : L'appareil formel de l'énonciation.

Pour ne prendre qu'un exemple, considérons la situation d'un père qui, excédé, crie à son enfant :

- Va voir là-bas si j'y suis!

Cette énonciation intime à l'enfant l'ordre d'aller « là-bas ». L'endroit en question ne peut être déterminé que par rapport à la position occupée par le père, le hic, l'« ici » du locuteur. Ainsi tout l'appareil déictique s'origine dans la situation spatio-temporelle du corps du locuteur qui fonctionne comme déictique non verbal relevant de l'intexte ${ }^{28}$. L'ordre, comme tout directif, suppose une action future de l'allocutaire. Ce futur est à l'aune du nunc, le présent de l'énonciation par le locuteur. Si l'enfant va "là-bas ", il ne pourra y voir son père qui, lui, n'ayant pas à obéir à l'ordre n'y sera pas et qui, de toute façon, ne peut ${ }^{29}$ y être au moment de l'énonciation! D'où l'inanité pragmatique de l'ordre que l'enfant ressent immédiatement et qui le conduit pertinemment à une seconde interprétation du directif, indirecte cette fois : l'impératif de ne plus l'importuner.

Ainsi l'appareil formel de l'énonciation détermine pragmatiquement les circonstances. Reste qu'il convient d'aller plus loin pour analyser praxéologiquement la situation.

28 Ce qu'avait bien vu Hegel : " L'ici est, par exemple, l'arbre. Je me retourne. .../... l'ici n'est pas un arbre, mais plutôt une maison ", La Phénoménologie de l'Esprit (1807), trad. fr. J. Hyppolite, Paris, Aubier, t. 1, 1998, $\$ 1$, p. 84.

29 Ce qui requiert la croyance d'arrière-plan selon laquelle le père n'est pas doué d'ubiquité. 


\section{Situation intersubjective}

Depuis Martin Buber ${ }^{30}$ les philosophes du dialogue ont coutume de séparer la personne qui se révèle dans et par le dialogue de son support individuel. Récusant cette scission idéaliste, nous inscrivons au contraire la personne qu'est l'interlocuteur dans sa concrétude individuelle. Le dialogue se déploie dans une situation interindividuelle mettant en relation (au moins) deux interlocuteurs. Au cours de la transaction intersubjective, ils se reconnaissent mutuellement [dimension psychologique (profil), sociologique (faces), institutionnelle (places), économique (statut), idéologique (croyances, religion, etc.), politique (affliation), etc.]. Selon le type de dialogue et ses enjeux intramondains, tel ou tel aspect de cette dimension interindividuelle joue un rôle plus ou moins important.

\section{Situation intramondaine}

Outre des personnes possédant leur individualité propre, les partenaires du dialogue sont aussi et d'abord des interactants partageant la même situation, identifiant ensemble un problème (Aufgabe) et lui apportant une solution en intervenant dans un micro-monde qu'ils construisent en commun.

Même si chacun a ses buts propres, les interactants s'entendent pour résoudre une tâche commune qui surgit de la situation partagée. Ils doivent alors négocier la stratégie à adopter et déterminer mutuellement les voies et moyens pour atteindre la fin acceptée. Ils coopèrent alors pour parvenir à la fin visée ${ }^{31}$. Le dire comme faire interactionnel est tributaire et dépend du faire transactionnel. La signification langagière n'est que l'écume d'un sens qui s'avère irrémédiablement transactionnel. Ce que Wittgenstein rappelle opportunément ainsi :

Ce que nous disons reçoit son sens du reste de nos actions ${ }^{32}$.

Pour prendre un premier exemple simple, si dans un échange survient l'énonciation :

- Paul et Marie ont la même voiture,

et si le cotexte ne lève pas l'ambiguïté, celle-ci l'est par la connaissance mutuelle de la situation :

30 Je et Tu (1923), trad. fr. G. Bianquis, Paris, Aubier, 1969, p. 99 : «La personne contemple son $s o i$, l'individu s'occupe de ce qui est sien; il dit : mon espèce, ma race, mon activité, mon génie. L'individu ne participe à aucune réalité et n'en conquiert aucune ". Voir aussi notre Introduction à la philosophie contemporaine du langage, Paris, A. Colin, 2010, \$ 6.2, p. 131.

31 Le concept de coopération a été clairement défini par Tadeusz Kotarbinski dans le Traité du travail efficace (1953), trad. fr. coord. par J.-L. Dumont, PU Franche-Comté, 2007, chap. 7. Comme tout concept, il est bipolaire : existent ainsi des coopérations positives ou négatives, voir notre Introduction à la philosophie contemporaine du langage, $\$ 4.1 .2$.

32 De la certitude, $\$ 229$. 
- Si Paul et Marie vivent ensemble, on peut supposer qu'ils partagent une même voiture (même signifie alors l'identité).

- Par contre, si ce sont deux amis qui vivent éloignés l'un de l'autre, il y a fort à parier qu'ils ont des voitures de même type (la relation est alors de simple similitude).

Considérons un second exemple supposant un couvreur qui dit à son apprenti :

- Il y a quatre tuiles dans le camion.

Abstraitement, cette énonciation a la forme d'une assertion susceptible d'être vraie ou fausse. Or, dans le camion il reste huit tuiles. L'assertion serait alors fausse. La considération de la situation praxéologique partagée par le couvreur et son apprenti impose plutôt d'interpréter l'énonciation comme un ordre indiquant où sont les tuiles et combien il faut en apporter. L'interacte construit dialogiquement passe de l'assertif apparent au directif effectif.

Dans le premier exemple, la considération des données situationnelles tranche l'ambivalence sémantique, dans le second elle détermine pragmatiquement la force illocutoire de l'acte de discours.

L'analyse praxéologique doit donc tenir compte des deux dimensions intersubjective et intramondaine de la situation pour rendre compte du sens et de la finalité du dialogue. Par exemple, dans une consultation médicale, la transaction intersubjective consiste à se co-constituer comme médecin et patient avec leurs caractéristiques propres et à s'accorder sur la finalité thérapeutique du dialogue qui passe par le diagnostic et la proposition de traitement ${ }^{33}$.

\section{L'arrière-plan actionnel}

On pourrait croire que l'on a enfin atteint le terme de l'analyse. Il n'en est rien car la situation intersubjective et intramondaine qui lie les partenaires et co-actants s'avère tributaire d'un arrière-plan actionnel inscrit dans une forme de vie assurant l'accord, plus ou moins institutionnalisé, sur le sens et la finalité actionnels de l'échange langagier ${ }^{34}$.

Si la situation lie les deux interlocuteurs et co-actants dans leur singularité datée et située, et si elle relève du niveau représentationnel en ce qu'elle peut faire l'objet d'une objectivation et d'une analyse, l'arrière-plan est plus

33 Pour un examen plus précis du dialogue médecin-malade, voir notre article : « Les niveaux d'analyse des phénomènes communicationnels : sémantique, pragmatique et praxéologique ", Analyse et simulation des conversations. De la théorie des actes de discours aux systèmes multiagents, B. Moulin et B. Chaib-draa (éds), Lyon, L'Interdisciplinaire, 1999, p. 101-131.

34 Sur cette dimension praxéologique ultime, voir notre Discours et vérité, 2009, chap. X : " Discours et action », p. 177-199. 
large et relève de la manière dont dans une société et une "culture " données un groupe humain régit et institutionnalise les rapports des Hommes entre eux et avec la Nature. Cet arrière-plan, de nature anthropologique, n'est plus représentationnel, mais bien actionnel et il caractérise les certitudes pratiques qui fournissent un réseau de prédispositions gouvernant aussi bien l'échange dialogique que les actions qui en découlent. De lui dépendent tropismes, aptitudes, dispositions, habitudes acquises et routines familières.

Considérons d'abord le rôle de l'arrière-plan dans la détermination de la signification. Ainsi l'énoncé austinien "Le chat est sur le paillasson " acquiert une signification sémantique quand on connaît le Français, mais son sens requiert absolument son inscription dans un arrière-plan situé et daté. Comme le rappelle Searle, reprenant Wittgenstein ${ }^{35}$, la référence au chat et au fait selon lequel il est sur le paillasson ne va pas de soi et engage des connaissances d'arrière-plan communes aux interlocuteurs et fournies non plus par l'énonciation elle-même, mais par la forme de vie partagée par les interlocuteurs :

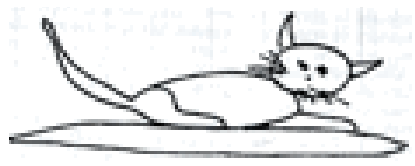

Supposons maintenant que le chat et le paillasson .../... flottent tous les deux dans l'espace intersidéral, voire dans une autre galaxie que la Voie lactée. Dans cette nouvelle situation, la scène serait aussi bien représentée si on regardait le dessin de biais ou à l'envers, puisqu'il n'existe pas de champ de gravitation relativement auquel l'un soit au dessus de l'autre. Le chat est-il encore sur le paillasson ? Et le champ de gravitation terrestre faisait-il partie des choses représentées sur notre dessin. ... /... La notion de sens littéral de la phrase « Le chat est sur le paillasson " n'a une application claire que si l'on fait quelques assomptions supplémentaires, dans le cas où chats et paillassons flottent librement dans l'espace intersidéral ${ }^{36}$.

On comprend alors aisément en quoi le dialogue effectif, dans ses deux dimensions inter- et transactionnelle, s'avère in fine tributaire d'une forme de vie, contingente et historiquement déterminée ${ }^{37}$ qui fournit le cadre praxéo-

35 Le concept d'arrière-plan (background) est d'origine wittgensteinienne : « Nous jugeons une action d'après son arrière-plan dans la vie humaine. .../... L'arrière-plan est le train de la vie ", Remarques sur la philosophie et la psychologie, trad. fr. G. Granel, Paris, Trans-Europe-Repress, 1998, \$\$ 624-625.

36 Sens et expression, trad. fr. J. Proust, Paris, Éd. de Minuit, 1982, chap. 5 : « le sens littéral ", p. 172. C'est la première thématisation searlienne du concept d'arrière-plan. Par la suite il lui accordera de plus en plus de place.

37 On peut envisager une Urlebensform qui fournirait les déterminants praxéologiques universels, mais ceux-ci ne seraient qu'abstractions par rapport à leur devenir historique et contingent. Reste que "La manière d'agir commune aux hommes est le système de référence au moyen duquel nous interprétons une langue qui nous est étrangère ", Wittgenstein, Investigations philosophiques, $\$ 206$, p. 203. 
logique ultime du sens des dires et de la finalité des actions ${ }^{38}$. Pour ne prendre qu'un exemple, considérons l'intentionnalité des agents. Une telle intentionnalité est attribuée à chaque agent qui devient moralement et juridiquement responsable de ses actes. Mais comment en rendre compte philosophiquement ? Une approche phénoménologique consiste à l'assigner en propre au sujet transcendantal. C'est ce que fait encore Searle en adoptant une conception du sujet héritée de Descartes revue par Brentano ${ }^{39}$. Il importe au contraire de récuser cette interprétation idéaliste pour affirmer le primat d'une intentionnalité qui trouve son origine non dans un sujet autonome, mais bien dans les potentialités fournies par l'arrière-plan socio-historique :

Lintention est inhérente à la situation, aux coutumes et aux institutions humaines. S'il n'y avait une technique du jeu d'échec, je ne pourrais avoir l'intention de jouer une partie d'échec ${ }^{40}$.

Ce même arrière-plan, où s'enracine l'intentionnalité individuelle, est en même temps source de la compréhension mutuelle ${ }^{41}$ ainsi que la possibilité d'entente aussi bien dialogiques qu'actionnelles en ce qu'aux potentialités offertes au locuteur-agent répondent réciproquement les attentes de l'allocutaire co-agent.

Sans pouvoir développer ce point crucial, ajoutons simplement que cette possibilité de compréhension et d'entente mutuelle dépend d'un jeu inférentiel mobilisant les données d'arrière-plan. Interviennent outre la classique déduction, l'abduction qui autorise des hypothèses sur les causes ${ }^{42}$. Prenons un rapide exemple d'un tel jeu inférentiel au cœur d'un échange prosaïque. Supposons le dialogue suivant entre une épouse encore couchée et son mari qui se lève pour aller au travail :

1 - Quel temps fait-il ?

2 - La rue est mouillée.

$3-$ Il a plu?

$4-$ Oui.

5 - Prends ton parapluie.

38 Cet arrière-plan est proche de ce que, dans une perspective sémiotique et interprétative, Eco appelle « encyclopédie », voir Sémiotique et philosophie du langage, trad. fr. M. Bouzaher, Paris, PUF, 1988, chap. 2. Il est aussi thématisé en éthnométhodologie sous le terme de common sense, voir Garfinkel, Studies in Ethnomethodology, Prentice-Hall, Inc., Englewood Cliffs, New Jersey, 1967, Essay 2.

39 L'Intentionalité, trad. fr. Cl. Pichevin, Paris, Éd. de Minuit, 1997. Chez Searle existe une tension irrésolue entre sa définition «phénoménologique » du sujet et son adoption du concept wittgensteinien d'un arrière-plan actionnel. Pour une approche plus pertinente de l'agent, voir Ricour, Soi-même comme un autre, Paris, Seuil, Points, n 330, 1990.

40 Wittgenstein, Investigations philosophiques, $\$ 337$.

41 Pour une analyse des modes de compréhension de l'action, voir Rémi Clot-Goudard, L'Explication ordinaire des actions humaines, Montreuil, Ithaque, 2015.

42 Sur la définition de l'abduction, vide notre "Singularité logique et praxéologique de l'abduction ", L'abduction, Recherches sur la philosophie et le langage, Paris, Vrin, 2017. 
En 2 le mari communique un fait qui vaut comme réponse à la question de l'épouse. La question de celle-ci en 3 propose le résultat d'une abduction immédiate qui s'appuie sur l'expérience courante. La réponse du mari en 4 valide cette proposition d'explication causale. Le conseil en 5 résulte d'une autre abduction, à savoir que s'il a plu, le temps a changé (la dépression est arrivée) et donc qu'il peut repleuvoir. D'une telle abduction suit par déduction ("S'il pleut, prendre son parapluie ») la conséquence pratique qu'il est bon de prendre un parapluie.

Pour tenir compte du rôle de l'arrière-plan inscrit dans une forme de vie, il convient donc de compléter notre schéma ainsi :

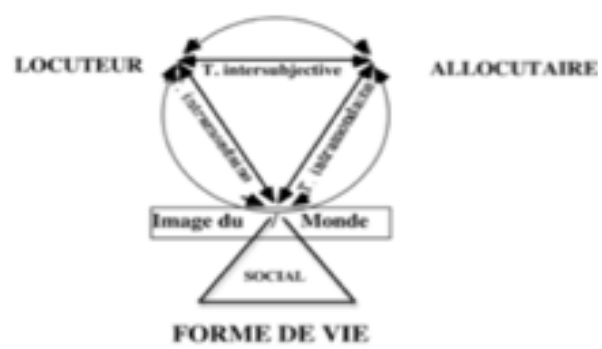

Fig. 4 : La forme de vie comme condition du dialogue.

L'image du monde co-construite relève alors d'une dimension proprement actionnelle et non plus seulement représentationnelle :

Le terme, ce n'est pas que certaines propositions nous apparaissent à l'évidence comme vraies immédiatement, donc ce n'est pas, de notre part, une sorte de voir ; le terme, c'est notre action qui se trouve à la base du jeu de langage ${ }^{43}$.

\section{Du perlocutoire}

La considération du contexte lato sensu tel que nous venons de le découper joue un rôle non seulement dans le choix de l'interprétation sémantique et de la détermination pragmatique de la force illocutoire des énonciations, mais surtout elle fournit les informations permettant d'appréhender le perlocutoire. On sait que pour Austin le perlocutoire porte sur les objectifs attendus de la part $\mathrm{du}$ locuteur et réciproquement sur les effets produits sur l'auditeur ${ }^{44}$. Or ce niveau n'est manifestement pas conventionnel et donc non prévisible a priori. Il est pourtant celui où se jouent les finalités praxéologiques du dialogue. Or, la prise en compte fine de la situation et de l'arrière-plan permet, sinon de prévoir, du moins d'expliquer les effets perlocutoires produits. Ce sujet important demanderait de longs développements. Considérons un simple exemple.

43 Wittgenstein, De la Certitude, $\$ 204$.

44 Quand dire, c'est faire, Neuvième conf. : " Les actes perlocutoires [sont] les actes que nous provoquons ou accomplissons par le fait de dire une chose ", p. 119. Ce sont les conséquences, attendues ou non, du fait de dire sur l'auditeur. 
Soit au cours d'un dialogue l'énonciation :

- T'as d'beaux yeux, tu sais!

Abstraitement, il s'agit d'un expressif qui énonce un jugement favorable à propos des yeux de l'allocutaire. Le problème est que (malheureusement!) son effet perlocutoire n'est pas automatique. Mais il n'est pas pour autant aléatoire et arbitraire en ce que l'on peut soupçonner quelques conditions de sa réussite. Sans développer l'analyse, examinons certaines d'entre elles.

- L'adresse initiale « $T$ ' as » instaure une relation de familiarité avec l'allocutaire (tutoiement plus allitération). Est en jeu alors la transaction intersubjective entre allocutaires. Si le contexte dialogique (stricto sensu) n’a pas instauré une telle relation, l'énonciation a de fortes chances d'être reçue comme une provocation et d'engendrer une violente réaction de rejet.

- Le thème "d'beaux yeux " doit être pertinent, c'est-à-dire adapté à un trait saillant de l'allocutaire. Supposez que celui-ci n'ait pas de beaux yeux (ou du moins des yeux que l'on ne peut tenir pour beaux), l'effet sera catastrophique, le compliment virera à la dérision.

- «Tu sais » est une demande de partage de l'éloge qui a d'autant plus de chances de réussir que l'allocutaire est aussi convaincu de la justesse du jugement louangeur.

- Ce que nous transcrivons dans le texte du dialogue par un point d'exclamation (!) traduit ici l'intonation, la posture, la mimique, et surtout le regard, etc., signaux qui tous manifestent clairement l'expression du désir. Cette expression peut d'autant plus être bien reçue qu'elle demeure plus ou moins voilée, décente. Elle se veut une proposition (proposal) acceptable.

Dès lors, le sens et la finalité praxéologiques de cette énonciation relèvent d'un jeu de séduction qui s'ancre dans un arrière-plan partagé.

Nous venons ainsi de dégager quelques conditions praxéologiques de félicité de l'effet perlocutoire. Mais cette réussite ne peut être assurée que si d'autres conditions sont requises qui portent à la fois sur les niveaux intersubjectif et intramondain de la transaction poursuivie entre les interlocuteurs : sont-ils sentimentalement libres, ont-ils des raisons de s'apprécier mutuellement, l'expression du désir du locuteur est-elle susceptible de susciter le même désir chez l'allocutaire, etc. ? Dans le meilleur des cas, on pourra avoir le dialogue suivant :

- T'as d'beaux yeux, tu sais [ton tendre]!

[Communion des regards]

- Embrassez-moi!

[Baiser]

- Nelly!

- Embrasse-moi encore ${ }^{45}$ !

45 Le Quai des brumes, 1938, film de Marcel Carné, dialogue entre Jean Gabin et Michelle Morgan 


\section{Le renversement praxéologique}

Adoptant une perspective actionnelle et dialogique nous avons procédé à une analyse conceptuelle conduisant à distinguer progressivement texte, cotexte, intexte, contexte stricto sensu, situation et arrière-plan. Chacun de ces éléments fournit les informations indispensables pour assigner signification, sens et finalité à l'échange dialogique. Méthodologiquement, cela témoigne d'une approche stratifiée qui successivement considère les aspects sémantique, pragmatique et praxéologique de tout dialogue effectif. Pareille démarche suppose deux engagements philosophiques cruciaux en matière d'étude des phénomènes langagiers.

- Le premier réside dans le refus résolu de la clôture communicationnelle trop souvent admise par les philosophes du langage et les théoriciens de la communication. L'idée est fort simple : le dialogue, la communication n'ont pas leur fin en eux-mêmes. Sauf exception pathologique, on ne parle pas pour parler, mais pour agir ensemble et résoudre un problème commun. En résulte qu'il convient d'articuler analyse pragmatique et déterminations praxéologiques. On ne saurait donc vouloir séparer agir communicationnel et agir stratégique ${ }^{46}$, distinguer la personne comme interlocuteur purement rationnel de son substrat individuel mû par des besoins, intérêts, etc., tenus pour perturbateurs $^{47}$, ou pire, comme le fait Searle, exclure de l'approche théorique le niveau perlocutoire des actes de discours au prétexte qu'il n'est pas conventionnalisable ${ }^{48}$.

- Le second engagement consiste à ne pas confondre ordre d'exposition et ordre théorique. La succession sémantique, pragmatique, praxéologique reste purement pédagogique en ce que ces divers niveaux s'interpénètrent en fait ${ }^{49}$ et surtout en ce qu'ils doivent être inversés. L'approche idéaliste habituelle de la communication consiste sinon à récuser le niveau praxéologique, du moins à en minimiser autant que possible le rôle. Il convient au contraire

écrit par Jacques Prévert. L’analyse de tout le dialogue du film confirmerait aisément que toutes les conditions praxéologiques de félicité sont remplies!

46 Ce que fait pourtant Habermas à des fins fondationnelles, voir notre «Dialogue \& praxis, le cas Habermas ». Langage et politique, B. Geay et B. Ambroise (éds), Lille, Presses universitaires du Septentrion, 2016.

47 Voir Francis Jacques qui sépare nettement la personne de son substrat individuel, Différence et subjectivité, Anthropologie d'un point de vue relationnel, Paris, Aubier-Montaigne, 1982, chap. 3 , $\$ 2$, p. 149-152.

48 Voir notre article "Relire Austin ", La philosophie du langage ordinaire. Histoire et actualité de la philosophie oxonienne, B. Ambroise et S. Laugier (éds), Hildesheim, Georg Olms Verlag, 2011, $\$ 1.2 .2$, «l'éviction du perlocutoire".

49 Rappelons que la distinction entre texte et intexte est purement technique et n'a qu'une valeur analytique. 
de se souvenir qu'Im Anfang war die Tat ${ }^{50}$ et donc d'opérer un renversement praxéologique $e^{51}$ qui situe la source du sens et de la finalité du dialogue dans son enracinement actionnel.

Reste un dernier point que nous ne pouvons qu'évoquer ici, à savoir que par leur complexité spécifique les phénomènes dialogiques et communicationnels requièrent un traitement résolument indisciplinaire ${ }^{52}$ faisant appel pour tels et tels aspects à la philosophie du langage (ordinaire ou non), la linguistique, la sémiotique, la logique, l'informatique, la psychologie populaire, la sociologie, l'ethnométhodologie et l'anthropologie.

\author{
Denis Vernant \\ EA 3699 Laboratoire Philosophie \\ Pratiques et langages \\ Denis.Vernant@univ-grenoble-alpes.fr
}

\begin{abstract}
Résumé
Nous prenons pour objet le discours en tant qu'activité dialogique conjointe et processus imprévisible co-construit par au moins deux interlocuteurs. Analyser cette praxis dans ses dimensions sémantique, pragmatique et praxéologique nous conduit à distinguer le texte ; le cotexte ; l'intexte ; le contexte proprement dit ; la situation ; et enfin l'arrière-plan qui tous, à chaque niveau, fournissent les données indispensables à l'établissement d'une compréhension et d'une communication dialogique.
\end{abstract}

\title{
Mots-clés
}

Discours, dialogue, texte, cotexte, intexte, contexte, situation, arrière-plan.

\begin{abstract}
We take for object the speech as joint activity and unpredictable process co-built by at least two interlocutors. By analyzing this praxis in its semantic, pragmatic and praxeological dimensions we shall be led to distinguish the text; the cotext; the intext; the context stricto sensu; the situation; and finally the background, all determinations which, at each level, supply the essential data to the construction of a understanding and a dialogical communication.
\end{abstract}

\section{Keywords}

Speech, dialogue, text, cotext, intext, context, situation, background.

50 Goethe, Faust, I, scène 3, cité par Wittgenstein, De la certitude, $\$ 401$.

51 Pour une application à l'analyse de la fable Le corbeau et le renard, voir notre article "Le renversement praxéologique ou l'intelligence du Renard ", Anna Krol (éd.), Le langage comme action/l'action par le langage, Recherches sur la philosophie et le langage, $n^{\circ} 31$, Paris, Vrin, 2014.

52 Voir notre article "La dialectique indisciplinaire en sciences humaines ", Bulletin du Centre d'Études Médiévales d'Auxerre, BUCEMA, nº 17.2, 2013. 